\title{
LTE-U based Train to Train Communication System in CBTC: System Desin and Reliability Analysis
}

\author{
Hao Liang $\mathbb{D}^{1}{ }^{1}$ Hongli Zhao $\mathbb{D}^{2},{ }^{2}$ Shuo Wang $\mathbb{D}^{1},{ }^{1}$ and Yong Zhang $\mathbb{D}^{1}$ \\ ${ }^{1}$ School of Electronics and Information, Beijing Jiaotong University, 100044 Beijing, China \\ ${ }^{2}$ State Key Laboratory of Rail Traffic Control and Safety, Beijing Jiaotong University, 100044 Beijing, China \\ Correspondence should be addressed to Hongli Zhao; hlzhao@bjtu.edu.cn
}

Received 23 August 2020; Revised 8 December 2020; Accepted 14 December 2020; Published 31 December 2020

Academic Editor: Changqing Luo

Copyright (c) 2020 Hao Liang et al. This is an open access article distributed under the Creative Commons Attribution License, which permits unrestricted use, distribution, and reproduction in any medium, provided the original work is properly cited.

Communication-Based Train Control (CBTC) system is a critical signal system to ensure rail transit's safe operation. Compared with the train-ground CBTC system, the train control system based on train-to-train (T2T) communication has the advantages of fast response speed, simple structure, and low operating cost. As the core part of the train control system based on T2T communication, the reliability of the data communication system (DCS) is of great significance to ensure the train's safe and efficient operation. According to the T2T communication system requirements, this paper adopts Long-Term EvolutionUnlicensed (LTE-U) technology to design the DCS structure and establishes the reliability model of the communication system based on Deterministic and Stochastic Petri Nets (DSPN). Based on testing the real line's communication performance parameters, the DSPN model is simulated and solved by $\pi$-tool, and the reliability index of the system is obtained. The research results show that the LTE-U-based T2T communication system designed in this paper meets the train control system's needs for communication transmission. This paper's reliability evaluation method can complete the reliability modeling of train control DCS based on T2T communication. The research in this paper will provide a strong practical and theoretical basis for the design and optimization of train control DCS based on T2T communication.

\section{Introduction}

Today, the CBTC system has been widely used in urban rail transit systems [1]. The existing CBTC system is technically very mature. However, there are still certain shortcomings: the system structure is involved, which leads to too many interfaces between subsystems; the information transmission process is cumbersome, and the on-board equipment cannot directly obtain relevant information, which limits the increase in train speed; the system consists of more equipment, resulting in higher system construction and operating costs, which is not conducive to the large-scale development of urban rail transit [2].

As an improvement of the train control system based on train-to-ground communication, the train control system based on T2T communication has many advantages: simple structure, short transmission time, strong system flexibility, and low operating cost. Therefore, train control systems based on T2T communication have become an important development direction. Alstom implemented a streamlined CBTC system on line 1 of Lille, France, and first proposed a train control system based on T2T communication. There is no operating line of the train control system based on T2T communication in China, but there have been many related studies. Lin and others introduced T2T communication technology based on the existing train control system to achieve train collision protection, designed the overall architecture of the system, and analyzed the simplified system through the reliability block diagram [3]. Guan et al. conducted a series of detections on the T2T communication channel and investigated the influence of train operation on the communication channel [4]. Liu compared the new train control system with the traditional CBTC system in terms of structure, performance, maintenance, and backup mode and obtained the system [5]. These research results provide basic theoretical knowledge for the design of the train control DCS based on T2T communication. However, the existing research focuses on the performance of the communication 
system, and there has not yet been comprehensive systematic research on the reliability of $\mathrm{T} 2 \mathrm{~T}$ communication.

The train control DCS based on T2T communication reduces the system coupling. It simplifies the train communication process, thereby significantly reducing the DCS complexity and improving the system's efficiency. Also, the wireless communication technology used in the DCS has a more significant impact on the data transmission process between train control subsystems related to the quality of communication. Compared with wireless local area network (WLAN) technology, LTE technology based on the $1.8 \mathrm{GHz}$ licensed frequency band has strong anti-interference ability, complete mobility management function, high transmission rate, and low system delay. Now, it is successfully applied to urban rail transit [6]. However, with the advancement of smart urban rail applications, the contradiction between insufficient authorized spectrum resources and urban rail data transmission requirements is becoming more dangerous. As a supplementary technology to LTE, LTE-U aggregates authorized frequency band and unlicensed frequency band resources and has many advantages in licensed frequency bands. Simultaneously, the use of unlicensed frequency band resources can effectively alleviate the pressure of tight spectrum resources to meet the future development needs of urban rail transit.

LTE-U technology was first proposed at the 3GPP RAN62 summit in 2013, and 3GPP introduced it as a critical technology in LTE R13 [7]. Qing analyzed the technical advantages of LTE-U in applying urban rail transit through the professional research of LTE-U [8]. Bai researched the application of LTE-U in the urban rail transit vehicleground communication system, and its wireless resource scheduling management and mobility management algorithms [9]. At present, the research on the LTE-U DCS of urban rail transit is in its infancy, so it is of considerable significance to study the design and reliability of train DCS based on LTE-U.

The reliability of the DCS is of great significance for ensuring the safe and efficient operation of trains. The existing set of sophisticated research methods for the reliability research of the train control DCS based on train-to-ground communication is not entirely suitable for the reliability research of the train control system based on T2T communication. Besides, the existing model parameters in the reliability research of the DCS are almost all hypothetical, and the system performance parameters are not obtained from the real train control DCS. The main contributions of this paper are as follows:

(i) According to the requirements of the train control system based on T2T communication, using LTE$\mathrm{U}$ technology, a train control DCS based on T2T communication is designed

(ii) According to the technical characteristics of LTE-U, and the unique needs of train control, the reliability of train control DCS based on T2T communication is fully defined. In order to evaluate the reliability of the system, this paper focuses on the analysis of typical communication scenarios for $\mathrm{T} 2 \mathrm{~T}$ communication

(iii) Use DSPN to model T2T communication scenarios during train communication. Through the communication performance parameter test in the real line environment, the reliability of the $\mathrm{T} 2 \mathrm{~T}$ communication scene is obtained by using the $\pi$-tool simulation, and the influence of the communication limit time on the reliability of the communication system is studied

The rest of this paper is organized as follows. Section 2 introduces the design of the T2T communication system based on LTE-U. Section 3 proposes the reliability definition of train control DCS and analyzes and models typical scenarios. In Section 4, reliable performance parameters are obtained by testing the communication link of the actual line. Section 5 examines the reliability of the train control DCS based on the exact line performance parameters. Section 6 summarizes the paper.

\section{The Design of Train Control DCS}

2.1. LTE-U Wireless Data Communication Technology. In the early days, the DCS of CBTC used WLAN technology based on the IEEE802.11 series protocols to carry out two-way information transmission between train and ground [10]. Although the data wireless communication system based on WLAN can meet traditional urban rail transit requirements for availability, reliability, and safety, it can no longer meet the rapid development of urban rail transit operation requirements in the future. Its limitations mainly include limited operating speed and coverage. The distance is short, the link design is complicated, and wireless interference is dangerous. When multiple services are concurrent, it is impossible to schedule resources according to the priority [11].

LTE-M is an LTE system based on LTE wireless communication technology and customized according to the business needs of urban rail transit [12]. The LTE-M system fully considers the reliability and real-time requirements of urban rail transit business and combines the advantages of the LTE network to form a systematic and standardized solution, which makes up for the disadvantage of WLAN technology carrying CBTC service, and provides innovative technical support for the safe operation of urban rail transit. However, at present, LTE-M systems occupy the proprietary frequency band of $1785 \mathrm{MHz}-1805 \mathrm{MHz}$ [13] and are only allowed to use $20 \mathrm{MHz}$ bandwidth resources at most. At the same time, CBTC services adopt dual-network redundancy mode, and their single-network services usually need $5 \mathrm{MHz}$ bandwidth, so integrated bearer service networks often use $15 \mathrm{MHz}$ bandwidth resources at most. In addition, if the frequency transfer method is used to solve the interference problem of the public-private network, the wireless resources available in the urban rail transit system will be further reduced, and the comprehensive carrying service network is easy to block the network capacity. Reduce the performance of the communication system. Reduce the 
performance of the communication system. Therefore, new technology is needed to solve the problem of lack of spectrum resources to meet the growing needs of urban rail transit.

LTE- $U$ aggregates authorized band and unlicensed band resources through carrier aggregation (CA) technology and introduces it into urban rail transit DCS to supplement the limited spectrum resources of the LTE-M system. While the authorized band provides reliable and stable transmission, an unlicensed band provides high throughput transmission to meet the future development needs of urban rail transit. LTE-U inherits many advanced technologies of the LTE system and introduces its unique channel sharing strategy. LTE-U is the same as LTE-M in network architecture. Still, the frequency band of the RF work unit is different, including Evolved Packet Core (EPC), Base Band Unit (BBU), Radio Remote Unit (RRU), and User Equipment (UE).

At present, there are mainly three bands of unauthorized spectrum available for LTE- $\mathrm{U}$, namely, the $2.4 \mathrm{GHz}$ group for the industry, science, and medicine, the $5 \mathrm{GHz}$ band for unlicensed international information facilities, and the newly proposed 28 60 GHz millimeter-wave band [14]. Therefore, in the application of urban rail, LTE-U works in the $5 \mathrm{GHz}$ group, which will have abundant unauthorized spectrum resources and have a good application prospect. In order to realize the integrated service bearer with a high transmission rate and real-time requirements in the high-speed operation scenario, LTE technology is introduced into the $5.8 \mathrm{GHz}$ open frequency band, that is, LTE-U can achieve full highspeed data transmission of nonsecure information with higher security and environmental adaptability based on more precious frequency resources and higher spectrum efficiency [15].

As a new technology supported by 3GPP and MulteFire alliance, LTE-U technology has obvious technical advantages in urban rail transit applications, such as excellent mobility and handoff performance, small transmission delay, strong anti-interference ability, large coverage radius, good QoS guarantee, high security, and high reliability. Therefore, the application prospect of LTE-U technology in urban rail transit is broad.

2.2. Train Control System Based on T2T Communication. The train control system based on T2T communication simplifies the structure of train control system based on train-ground communication, reduces the ground equipment Zone Controller (ZC) and Computer Interlocking (CI), integrates its functions into on-board equipment, and adds Train Management Unit (TMU) and Object Controller (OC). The basic structure is shown in Figure 1.

In the process of train operation, the train runs according to the operation plan issued by the Automatic Train Supervision (ATS). When it is about to enter the next section of the line, the train actively sends a communication request to the TMU of the section, registers the identity information, and queries other train information in the line. TMU stores the information on the car and responds to the query of the train. According to the identity information obtained, the train establishes the communication between train and train and requests the location information of other trains. The train uses the speed measurement and positioning equipment and the transponder to determine the position of the train on the line, map it to the electronic map, and judge the logical relationship between the front and rear trains.

The on-board equipment calculates the endpoint of the Movement Authority (MA) through the interlocking module in the Vehicle On-Board Controller (VOBC) according to the driving plan, the status of the signal equipment beside the track, and the position information of the front train on the line. The Automatic Train Protection (ATP) module will calculate the train speed protection curve based on the MA, line information, temporary speed limit, and other information and update it in real time. Automatic Train Operation (ATO) completes the functions of train traction, speed adjustment, and automatic return under the supervision of ATP. At the same time, the train will check the status of the associated signal equipment in the route and request the control of the trackside equipment from the OC. OC responds to the query, returns the state of the device, and transfers control rights. When the train is about to clear from the line section, the train actively disconnects the communication connection with other trains on the line and cancels the train information in the TMU.

2.3. The Design of the T2T Communication System Based on $L T E-U$. Based on the network structure of the LTE-U communication system, combined with the structure of the train control system based on T2T communication, a train control DCS based on T2T communication using LTE-U communication technology is designed. The system structure is shown in Figure 2. ATS interconnects with the core network through the backbone network, and the core network is connected with the base station on the line. Trains within the signal coverage of the base station can communicate with ATS through wireless communication.

The specific functions of the network elements in the LTE- $U$ system are as follows:

(1) Core network EPC: EPC is a full IP packet core network, and all services are switched in the Packet Switch (PS) domain. EPC core network includes Mobility Management Entity (MME), Serving Gateway (SG), Packet Data Node Gateway (PGW), Home Subscriber Server (HSS), and other network elements. The primary function of MME is to control signaling transmission, authenticate and page the terminal, and realize mobility management such as handover and roaming. SGW routes and forwards packet data in the system. The functions of PGW include checking, filtering and filtering data, and interworking with networks outside the LTE system. HSS stores and manages user data and authentication data, including location information of mobile users

(2) Wireless access network E-UTRAN: wireless access network E-UTRAN is composed of a plurality of evolved NodeB (eNodeB). The communication network is connected wirelessly by eNodeB users. eNo$\mathrm{deB}$ has the functions of routing, establishing or 


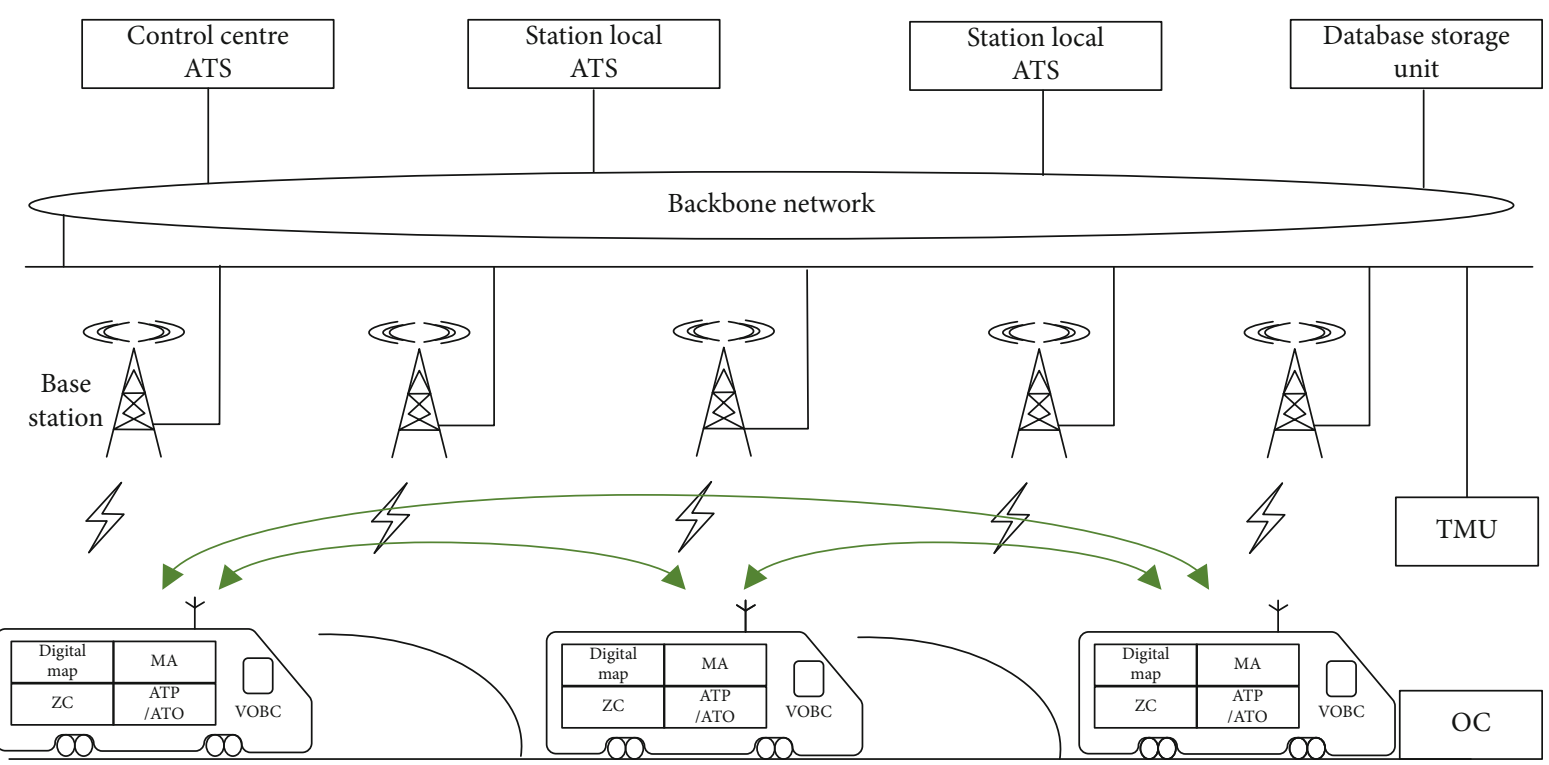

FIGURE 1: Basic structure diagram of the train control system based on T2T communication.

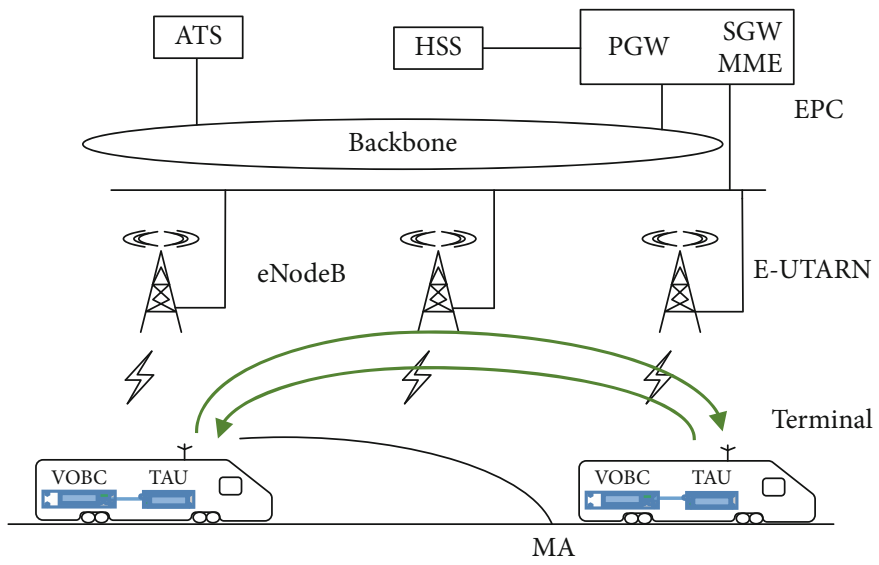

Figure 2: Train control DCS based on T2T communication.

releasing wireless links, mobility management, scheduling and allocation of wireless resources, bearer control, etc.

(3) Terminal: train installs train access unit (TAU) as the service data transmission equipment to realize wireless communication. The TAU is connected with the functional modules on the train to send the information that the train needs to upload through the antenna, and then, the information is transmitted to the ground equipment or other trains. At the same time, the TAU receives the information forwarded by the base station through the antenna and sends it to the train equipment after processing

\section{Reliability Definition and Scenario Models of Train Control DCS}

3.1. Data Transmission Flows of Train Control DCS Based on T2T Communication. Because the structure of train control
DCS based on LTE-U technology is different from that based on WLAN technology, the data communication flow is different from the previous communication system, so it is necessary to analyze the information transmission flow between train and train. As shown in Figure 3, the solid line is the data flow, and the dashed line is the signaling flow.

Curve 1 is the signaling flow channel after the train is electrified. When the train starts, TAU searches for cell signals, selects the cell, and triggers the random access process, ensuring communication links between terminals and networks. TAU sends a connection establishment request signal to the base station, allocating the radio resources for it. Simultaneously, through the S1-MME port, the base station sends the attachment request to the MME. After completing the TAU authentication, the MME sends the attached signal to the base station, and the base station sends the TAU capability information to the MME. Finally, the base station sends a security activation message, and after TAU activation, it sends configuration completion and attachment completion messages to the base station and MME. 
Curve 2 is the data flow channel of train communication under different base stations. The transmitting terminal sends messages to the base station through the empty port, and the base station forwards the messages to the SGW through the S1-U port. After the SGW inquiry, the messages are forwarded to the base station in the cell where the receiving terminal TAU is located and then to the receiving terminal TAU.

When the train reaches the overlap of base station signals, the service area has to be changed. Curve 3 is the channel of signaling flow based on X2 port switching. First, the source station sends measurement control down to TAU, which feeds back measurement reports. The source base station decides to switch based on the measurement report and then sends the switching request to the destination base station. After receiving the acknowledgment signal, the source base station sends configuration information to TAU. After the TAU configuration is completed, the source base station begins switching and sends the connection success information to the destination base station. The base station requests MME to update the data channel. TAU releases the cell resources under the source base station to complete the switch between stations.

3.2. Reliability Definition of Train Control DCS. The reliability of train control DCS affects the probability of a successful exchange of train information, so it is vital to use reasonable methods to evaluate its reliability. Based on the communication performance parameters, this paper defines the reliability of DCS from two links.

The first link is the reliability of data transmission. The reliability of data transmission is defined as the probability that the available information reaches the receiving end within a limited time. Both transmission delay and continuous packet loss affect the reliability of data transmission. According to the definition of reliability, as shown in Figure 4, (a) the packet delay $t 1$ is greater than the limit time $T$; then, the transmission of packet 1 is unreliable. Otherwise, the transfer of packet 2 is reliable; (b) when a continuous packet loss occurs, the duration of no data reception at the receiving end can be calculated according to the packet sending rate. If the term is greater than $T$, the data transmission is not reliable at this time.

The second link is the reliability of the communication scenario. During the train operation, communication processes such as data retransmission, switching between stations, and interrupt reconnection are required. Therefore, it is stipulated that the train needs to complete the communication process within the communication limit time. After the communication limit time is exceeded, the train will be in an unsafe operation state, and the reliability of the communication scenario is the probability that the train is in a safe state.

Since the existing reliability studies are mostly based on assumed parameters, in order to make the data communication system reliability research results more in line with the actual situation, the parameters of the model in this paper are derived from actual communication performance test results or the requirements of the communication system in

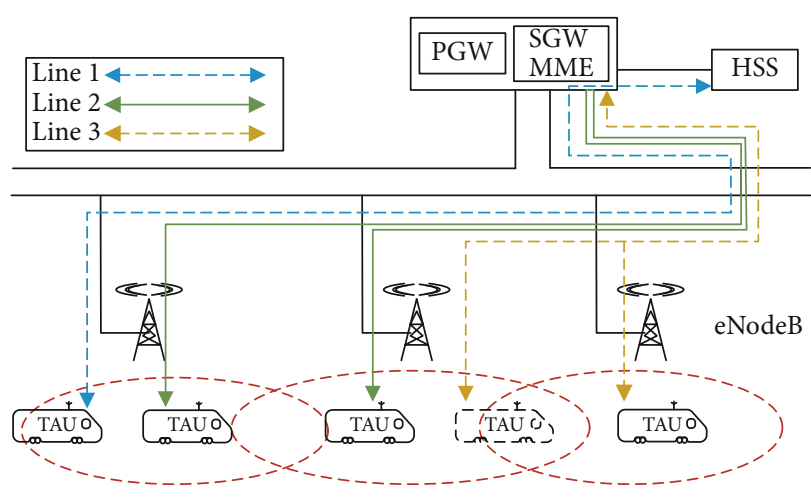

FIGURE 3: Signaling transmission flow and data transmission flow.

the urban rail transit communication specification, and the analysis results have higher practical significance.

3.3. Typical Scenario Analysis of T2T Communication. In the train control system based on T2T communication, the communication between trains is the primary demand, among which the communication scene of redundancy train access unit and roaming switching communication scene are typical communication scenes in the process of train operation.

\subsubsection{Communication Scenarios of Redundancy Train Access} Units. T2T communication is an important communication link in the process of train operation. However, the channel environment changes caused by geographical location, weather conditions, and other factors will reduce the communication quality, affect the real time and availability of the data received by the train, and then affect the regular operation of the train and the passenger's riding experience. In the existing research, it is a standard method to improve communication reliability using equipment redundancy. Therefore, the communication scenario of the redundant train access unit is typical in the T2T communication scenario.

As shown in Figure 5, two sets of train access units and on-board antennas are, respectively, configured at the head and tail ends of the train, and the two sets of communication equipment work independently and do not interfere with each other. Therefore, only one set of train access units can successfully receive reliable data within the specified time to ensure the train's regular operation. After receiving the information, the two sets of train access units process the information and obtain the available information required by onboard equipment, including train position information and obstacle information. Simultaneously, if the trackside equipment status and route information are obtained, the logic module of on-board equipment can calculate according to the relevant information to obtain the train operation permission terminal point. The emergency braking curve and automatic driving curve are calculated by the ATP and ATO logic module of on-board equipment, and the train is in normal operation.

3.3.2. Communication Scenarios of On-Board Terminal Handoff. Train cross-line operation is an inevitable requirement for the development of urban rail transit. It is necessary 


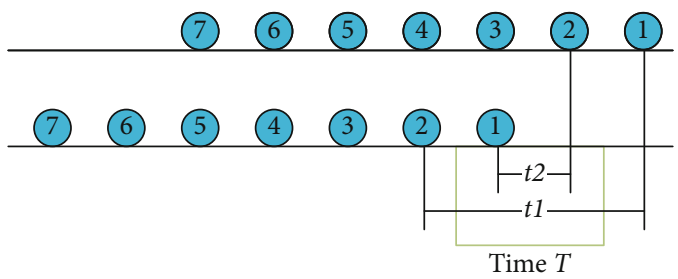

(a) Unreliable transmission caused by delay

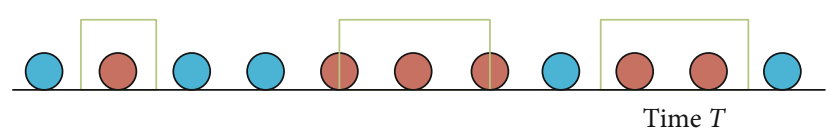

Received packet

Lost packet

FIgURE 4: The definition of data transmission reliability.

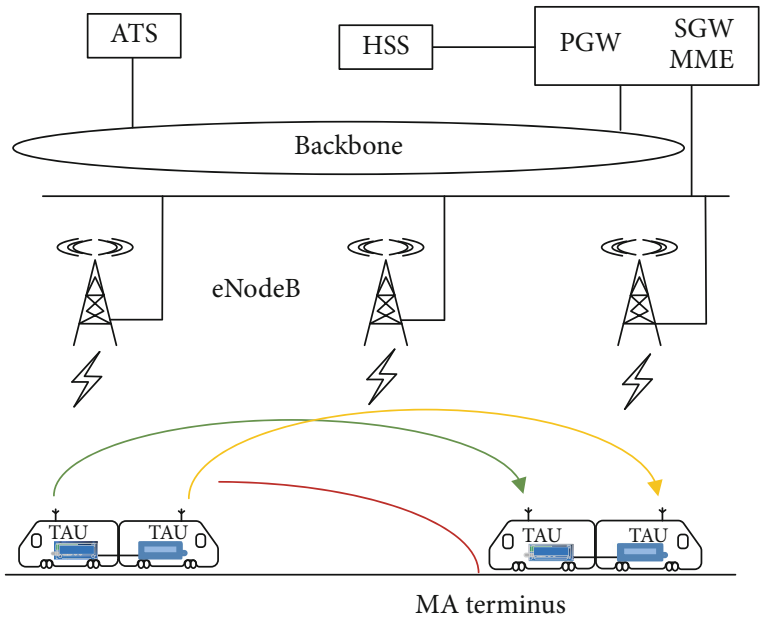

FIGURE 5: Communication scenario of redundancy train access units.

to ensure that the train can achieve on-board terminal roaming, realize interconnection, and save train resources. When the train is in the process of roaming switching, other trains on the line still need to communicate with each other. The roaming switching process has a more significant impact on the communication delay.

As shown in Figure 6, on the existing operation line, one set of communication equipment is used on the front line due to the construction planning. In contrast, another set of communication equipment is used on the follow-up line. When the train runs to the junction of two sets of core network equipment, it needs to carry out roaming switching.

As shown in Figure 7, when the train is roaming switching, the information sent by the ground equipment and other trains will be forwarded to the destination core network through the source core network and then to the base station of the switching train. After the roaming switching is completed, the train will obtain the information of the ground equipment and other trains on the line through the forwarding of the destination core network, which will increase large transmission delay. When the train crosses a line, the running interval of the line will change, and the train speed will be limited. To improve operation efficiency, it is necessary to quickly adjust the relevant parameters of train operation, so it is necessary to ensure the communication between trains.

\subsection{DSPN Models of T2T Communication Scenarios}

3.4.1. Deterministic and Stochastic Petri Net. The train control data DCS is a complex dynamic system, and its data communication has large randomness and high correlation with time. Therefore, this paper uses Deterministic and Stochastic Petri Net (DSPN) to model the train control DCS based on its data transmission characteristics.

A system of DSPN can be defined as a decimal group: $\Sigma$ $=\left(S, T, I, O, H, Y, K, \lambda, W, M_{0}\right)$. The store holds tokens, representing a network element of the system or a step in the process. In a DSPN, the maximum number of tokens that a store can hold can be set, the number of elements in the set $K$ for that store. Therefore, the state of the Petri net can be defined by the number of tokens in each store, as

$$
m=(\#(p 1), \#(p 2), \cdots, \#(p k))
$$

where $m$ is also called the identification vector of the Petri net. Each element is the number of tokens in the store $p$, and it must satisfy $M(p) \leq K(p)$. The set of $m$ is the set of system states.

In Petri nets, the triggering of changes represents events in the actual system, causing the system to transition from one state to another. Moreover, in stochastic Petri nets, transitions have implementation delays, and implementation delays are associated with a particular distribution, and the set of transition implementation rates is $\lambda$. In DSPN, according to different implementation delays, there are three changes:

(1) The implementation delay is zero. Such a transition is called an immediate transition, indicating a controlling role or a logical choice that does not require processing time

(2) The implementation delay is a random variable. This kind of vicissitude is one kind of time vicissitude, which is called exponential vicissitude. It indicates that the event takes a certain amount of time, and the distribution of time follows an exponential distribution: $F(t)=1-e^{-\lambda t} \lambda>0$

(3) The implementation delay is a definite value. This kind of transition is called fixed-time transition, which means the implementation delay of the transition corresponds to the processing time in the real system 


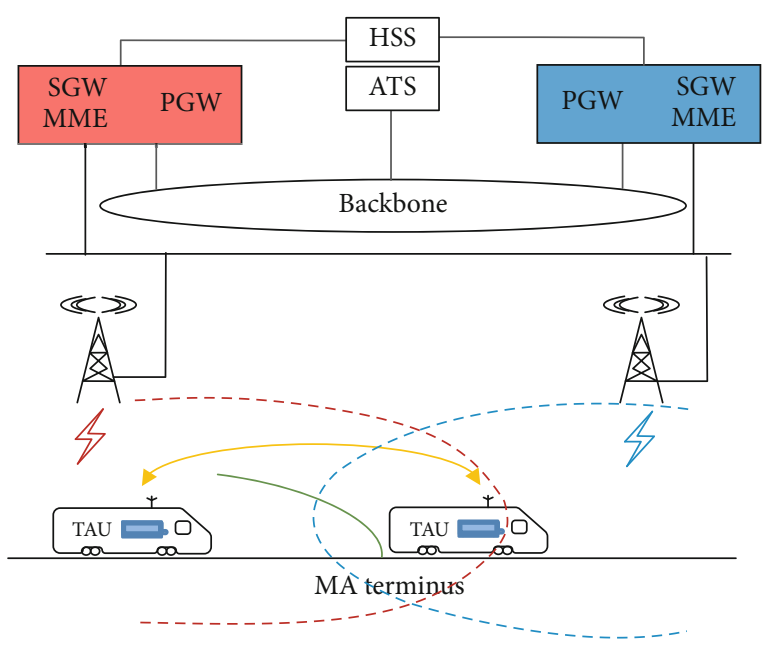

Figure 6: Train roaming communication scenario.

Besides, the Petri net visual simulation tool $\pi$-tool is used to establish a communication model and simulate [16-18]. The $\pi$-tool can realize the establishment of many kinds of Petri net models. It is a perfect modeling and simulation tool and has many advantages:

(i) Model building: the implementation rate of transitions in the software can be set to a variety of common distributions. For immediate transitions, the weight and priority of the transitions can be set. Ability to build structured models

(ii) Model analysis: the Petri net model can be deadlocked to detect system resource allocation and consumption. The reachability graph can be automatically generated according to the model to determine the state space and state transition of the system. Using Monte Carlo simulation analysis, the Petri net model with any transition can be analyzed to obtain the occupancy rate of the store, and a certain transition can be specifically observed to determine the time when it is first triggered

In the $\pi$-tool graphical interface, each element of the DSPN is graphically represented, as shown in Table 1.

3.4.2. Models of Communication Scenarios for Redundancy Train Access Units. The model is shown in Figure 8. First, the train is in the typical running state run, and then triggers transition GenMsg to start data transmission. At the same time, triggering transition trans, timer timer is started. This timer is the communication limit time for the train to complete an individual link. After that, the token is transferred to the store train0, which means that the information is transmitted to another train, triggering the transition eNo$d e B$, and the token is transferred to the store train, which means that the data is transmitted to the base station. At this time, there are two situations. The train is in the process of switching between stations or interrupting the reconnection process. The transition handover indicates that the train is performing the switching process. The stores HOX2TAU1.Msg and HOX2TAU2.Msg are the switching processes required by the two train access units. The transition interrupt means that the train's reconnection process is performed, and the stores AttachTAU1.connection and AttachTAU2.connection are the reconnection process required by the two train access units. This paper considers the most unfavorable situation: the handover and reconnection processes need to start from the initial signaling.

In this communication scenario, if both train access units need to switch between stations, and in the case where one train access unit switches successfully, the switching time of the other train access unit is no longer limited. The method for judging whether the reconnection of the two train access units is successful is the same as the handover. Only one of the train access units completes the reconnection process within the communication limit can be regarded as a successful reconnection. Taking the first train access unit successfully switching or reconnecting as an example, the switching or reconnecting submodel transfers the token to store success. At this time, through the limiting effect of the test arc, stores AttachTUA2.fail and HOX2TAU2.fail will obtain the cause the token generated by the transition failTAU2 that triggers the second train access unit to switch or reconnect the token in the submodel, and the model can start the next information transmission. However, if the timer has reached the communication limit time during the handover or reconnection, it is judged that the handover or reconnection is unsuccessful. At this time, all tokens in the submodel are cleared, and the system state changes from normal to danger until the next time the train successfully completes the handover or reconnection within the specified time; the system status changes to normal.

3.4.3. Models of Communication Scenarios of On-Board Terminal Handoff. Figure 9 shows the DSPN model. This is the top-level model. There are three bottom-level submodels under the top-level model, namely, the HOS1, Transmission, and TAUpd submodel. When the train runs, it generates information that needs to be sent to other trains, that is, the token is transferred from the store run to Msg. At this time, the transition trans is triggered, and the token is transferred to the timer timer, which limits the time of the communication process. At the same time, a token is transferred to the store train 0 and data transmission begins. The transition $e N o d e B$ trigger indicates that the data has been transmitted to the base station. In the most unfavorable situation, another train needs to complete all interactive processes of roaming switching.

First, the train performs the cross-core network switching process, and the token is transferred to the submodel HOS1 through the associated store HOS1.handover, and the store $P N . H O s u c c e s$ representing the completion of the switch in the submodel is associated with the HOsuccess store in the top model. After the submodel process is completed, the token is transferred to HOsuccess. Afterwards, the data is forwarded. The data is forwarded from the source core network to another core network. The store Transmission.data is associated with the store data in the submodel Transmission. In the submodel, after completing the process of data 


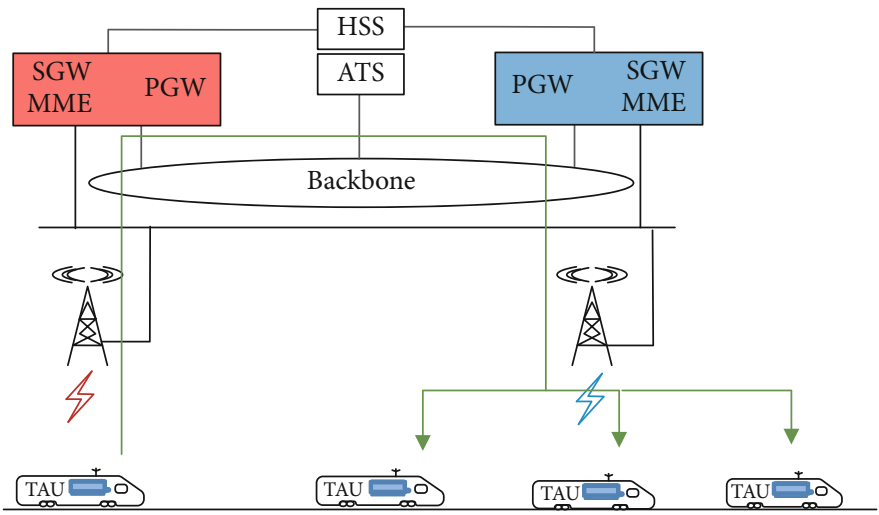

FIgURE 7: Data forwarding process.

TABLe 1: Graphic elements and their meanings of DSPN.

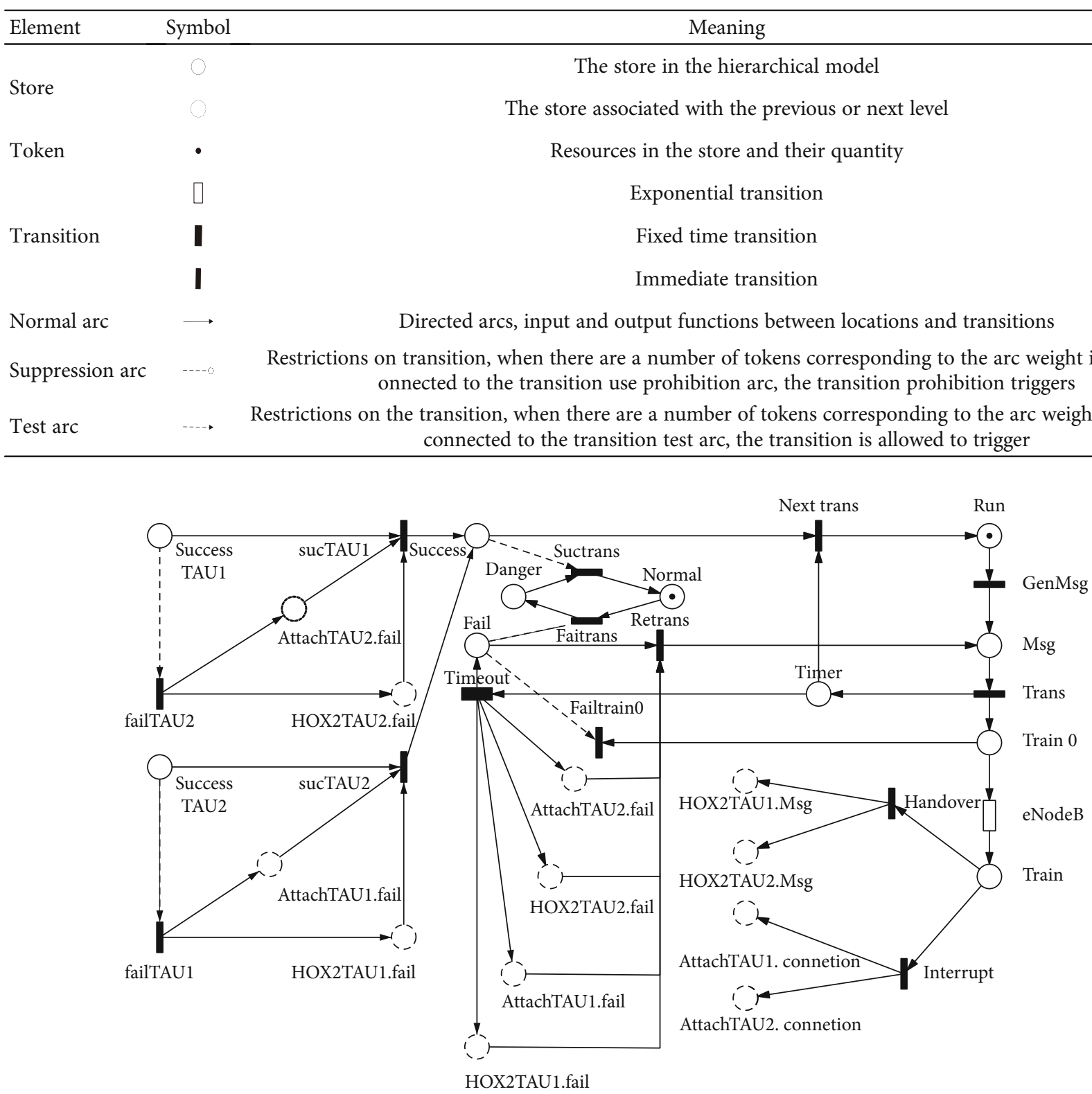

Figure 8: Models of communication scenarios for redundancy train access unit. 


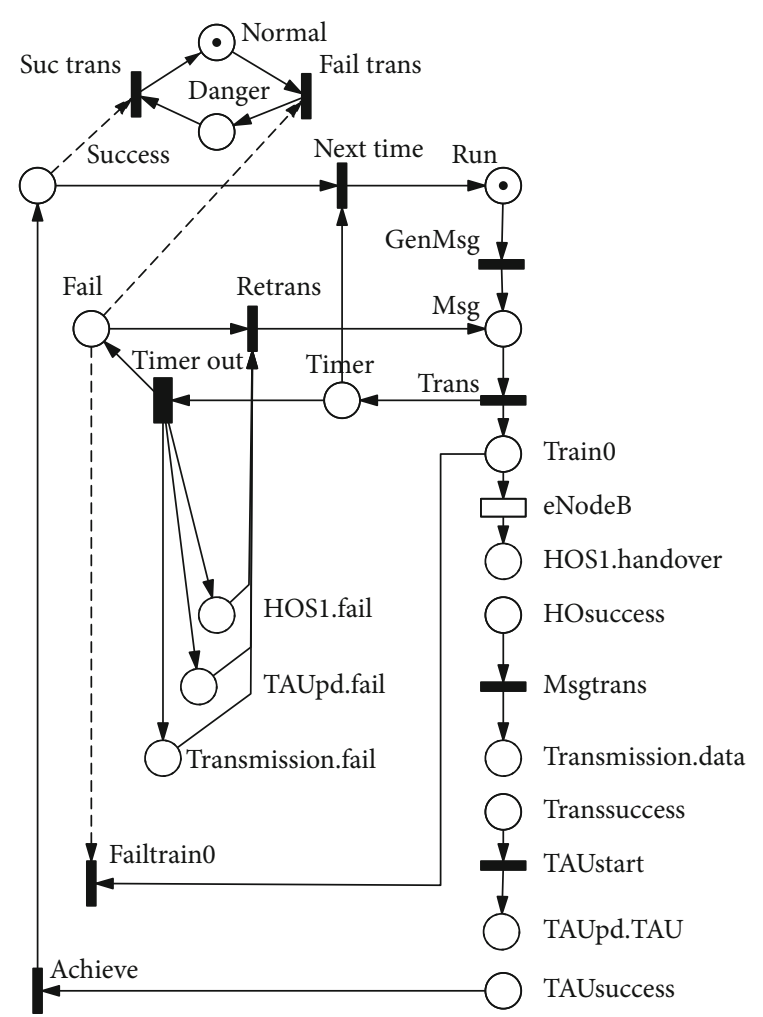

Figure 9: Models of communication scenarios of on-board terminal handoff.

transmission and retransmission, the token enters the store transsuccess in the top model through the associated store PN.transsuccess, indicating that the data forwarding process has been completed. Finally, by triggering the transition TAUstart, the tracking area update process begins. Similarly, the token enters the store TAU in the TAUpd submodel through the associated store TAUpd.TAU. After the token completes the tracking area update process in the signaling interaction sequence in the submodel, the token is transferred to the TAUsuccess store in the top model through the associated store PN.TAUsuccess. If the cross-core network handover process, data forwarding process, and tracking area update process are completed within the communication limit time, the next round of information transmission can begin. However, if the train fails to complete the roaming handover process when the timer reaches the communication limit time, indicating that the cross-core network handover fails, the top model triggers the timerout transition to cause orders to appear in the associated stores HO21.fail, TAUpd.fail, and Transmission.fail of the three submodels Card and then empty the token in the submodel. At the same time, the system state changes from normal to danger until the next successful roaming switching process; the system state changes to normal.

\section{Communication Performance Test of Train Control Data Link}

The transmission performance of the train control data link is very critical and affects the reliability of communication.
The existing reliability studies are mostly based on assumed performance parameters. Therefore, to make the research results of DCS reliability more in line with the actual situation, the model parameters in this paper need to be derived from actual communication performance test results or urban rail transit communications, the requirements for the communication system in the specification; such analysis results have higher practical significance. To determine the communication quality of the wireless link and obtain the transmission performance of the link during train operation, this paper is based on the actual operating line of Beijing metro line 15 to test the communication performance parameters of the link. The test section of the operating line is the 2 stations 1 section upstream section (including the station line) from Maquanying to Sunhe, a total of $3.3 \mathrm{~km}$, of which the $2.3 \mathrm{~km}$ section east of Maquanying Station is an underground tunnel, which is immediately adjacent to the viaduct, with a length of 1 kilometer to Sunhe Station, as shown in Figure 10.

The LTE-U system test in the test section of line 15 mainly includes three parts: communication room center equipment (EPC/switch, CCTV/PIS ground equipment), trackside base station equipment (8 sets), and on-board equipment (TAU/switch). Test networking is shown in Figure 11. Among them, base station 1 to base station 5 are covered by antennas with a distance of $400 \sim 450 \mathrm{~m}$, one side of base station 6 is covered by an antenna, a slotted waveguide covers the other side, and base station 7 and base station 8 are covered by the slotted waveguide.

First, test the data transmission delay and handover delay when the train is running. During the train operation, the train is within the signal coverage of a base station for a specified period. At this stage, the data transmission delay is only determined by the network environment and channel quality. When the train travels within the signal overlap range of the two base stations, the handover mechanism, handover between stations is required, and handover delay occurs. When the train is running on the line, seven handovers occur in a single journey, and seven handover delay data can be obtained. To obtain more handover delay data, the train runs back and forth between stations, and the handover delay is measured multiple times.

Figure 12 shows the delay and handover delay results obtained in a one-way test. At certain moments, the time delay will be significantly increased. The test time in the figure can be divided into eight stages, with a higher delay as the demarcation point. Each stage indicates that the train is within the signal coverage of an individual base station, corresponding to eight stages. For the signal coverage of each base station, a higher delay indicates that the train is at the junction of the base station's signal coverage. Therefore, there are seven handover delays in the figure. In the subsequent analysis, the delay and handover delay in all test data must be separately counted.

In order to avoid mutual influence when measuring the delay and packet loss at the same time, the delay and packet loss tests are carried out separately. To ensure sufficient data volume, the train runs multiple times to test and obtain packet loss data. If the software operation is interrupted, it 


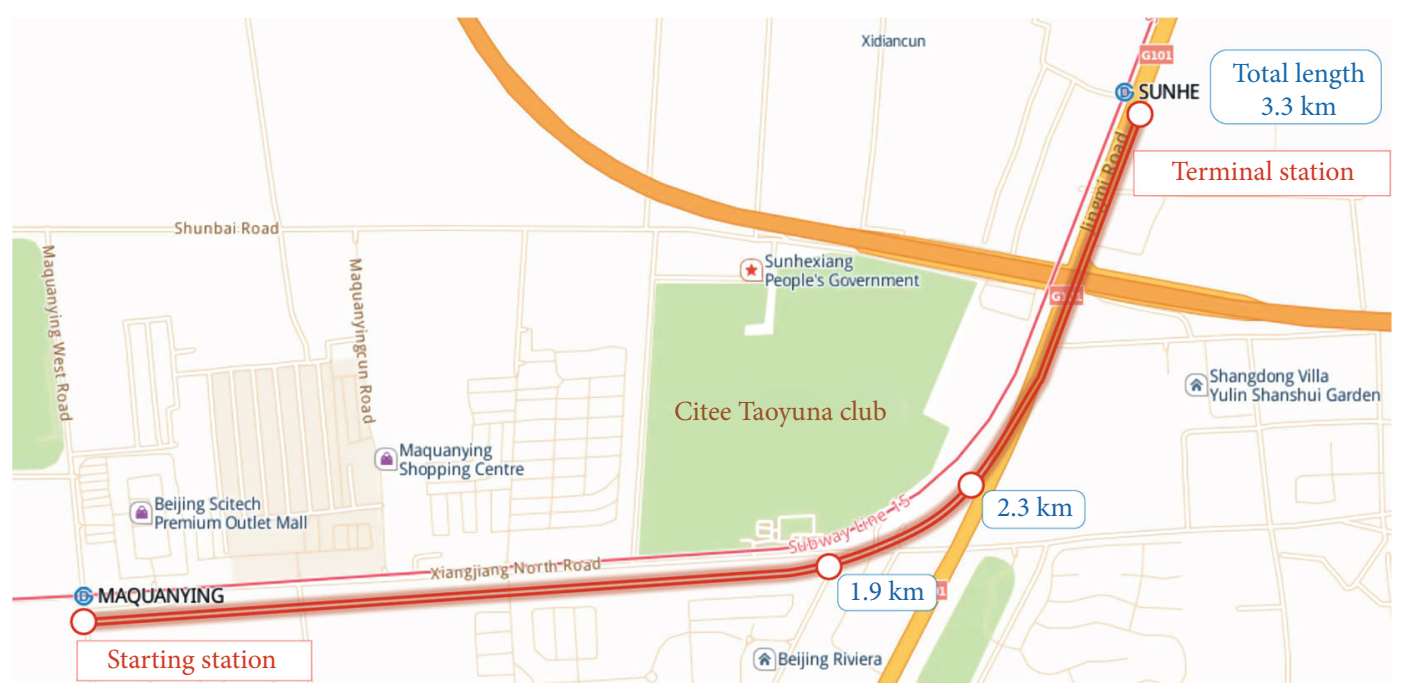

FIgUre 10: The test section of Beijing metro line 15.

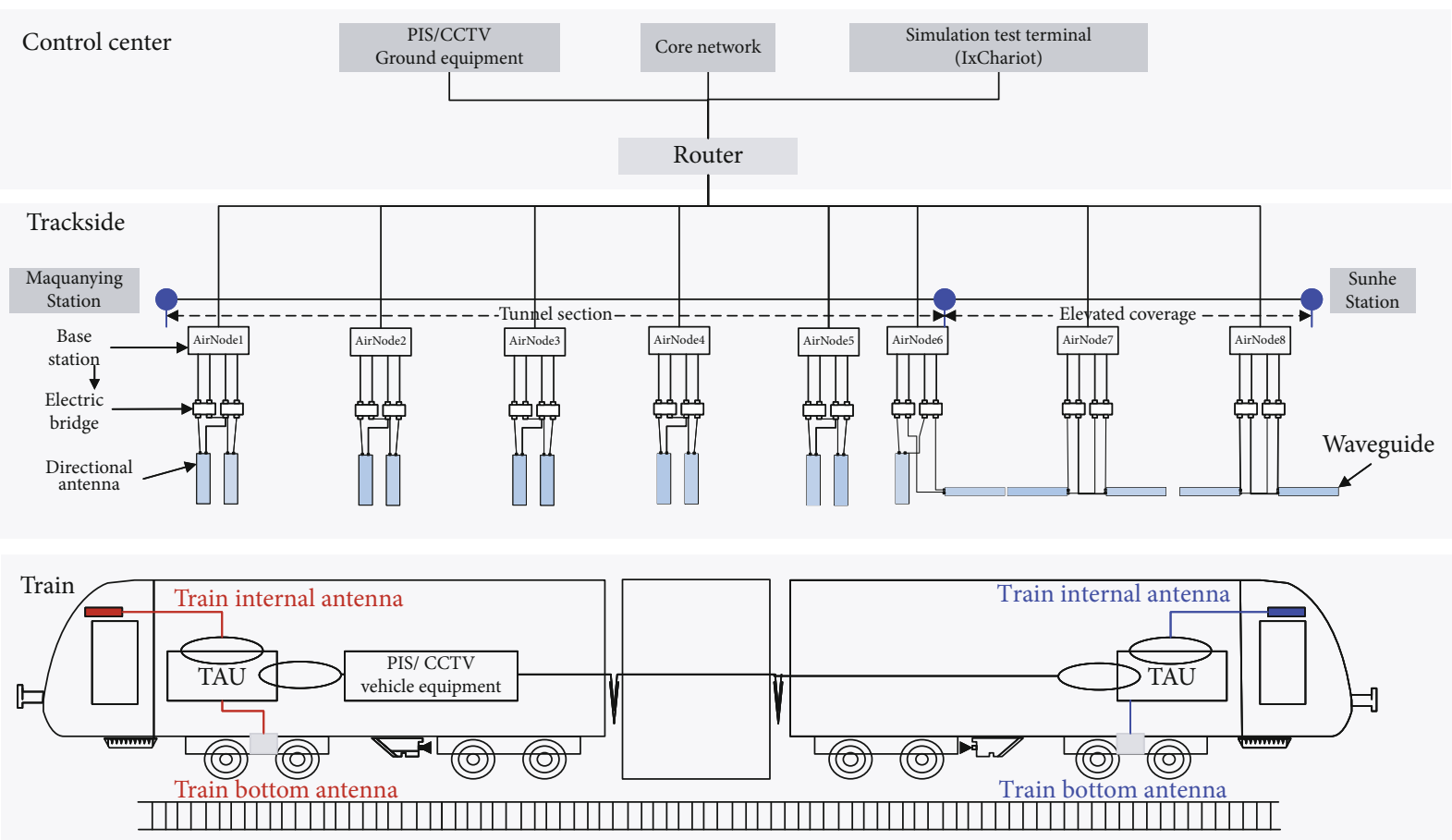

FIGURE 11: 5.8G system network structure diagram of line 15.

will affect the accuracy of the test results. Therefore, discard this set of data and perform it again to ensure the accuracy of the measured data. In the actual test, the packet loss rate test result is $0.497 \%$. Figure 13 shows the test result of continuous packet loss.

\section{The Reliability Analysis of Train Control DCS}

5.1. Reliability Analysis of Communication Scenarios for Redundant Train Access Units. This paper uses the Monte Carlo simulation method, and according to the reliability definition of the communication process in 3.1 , under the condition that the parameter value of the fixed transition timeout is different, the model is simulated. In practice, the parameter value of timeout represents the time limit of the signaling process performed on the train, from which the reliability under different communication time limits can be analyzed. The simulation obtains the reliability when the train is switched or reconnected separately under different timeout parameters.

According to the simulation results, as the timeout parameter value becomes more extensive, the communication scenario's reliability gradually increases. When the parameter value is $990 \mathrm{~ms}$, the reliability of the communication scenario 


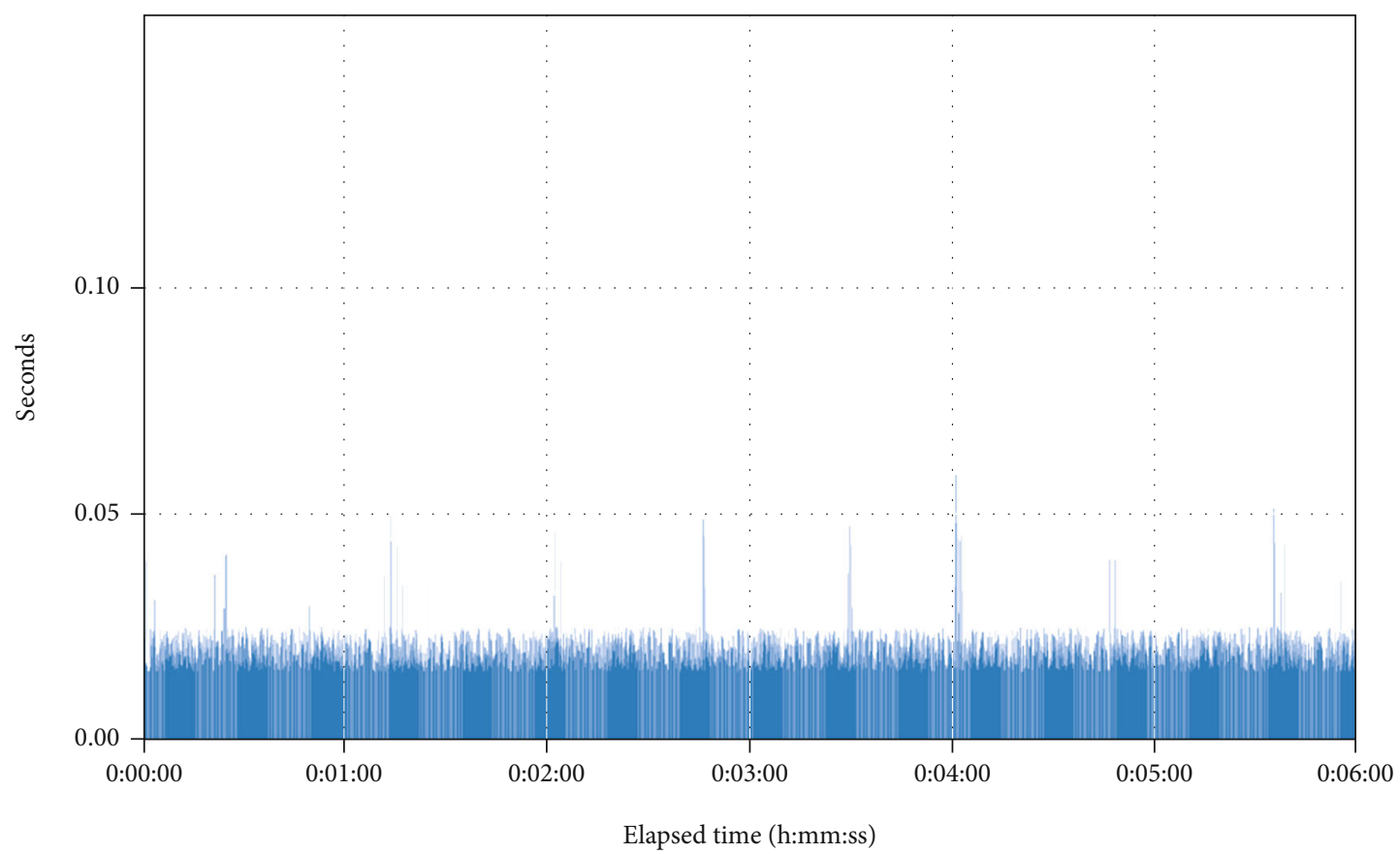

FIgure 12: Test results for transmission delay in the real environment.

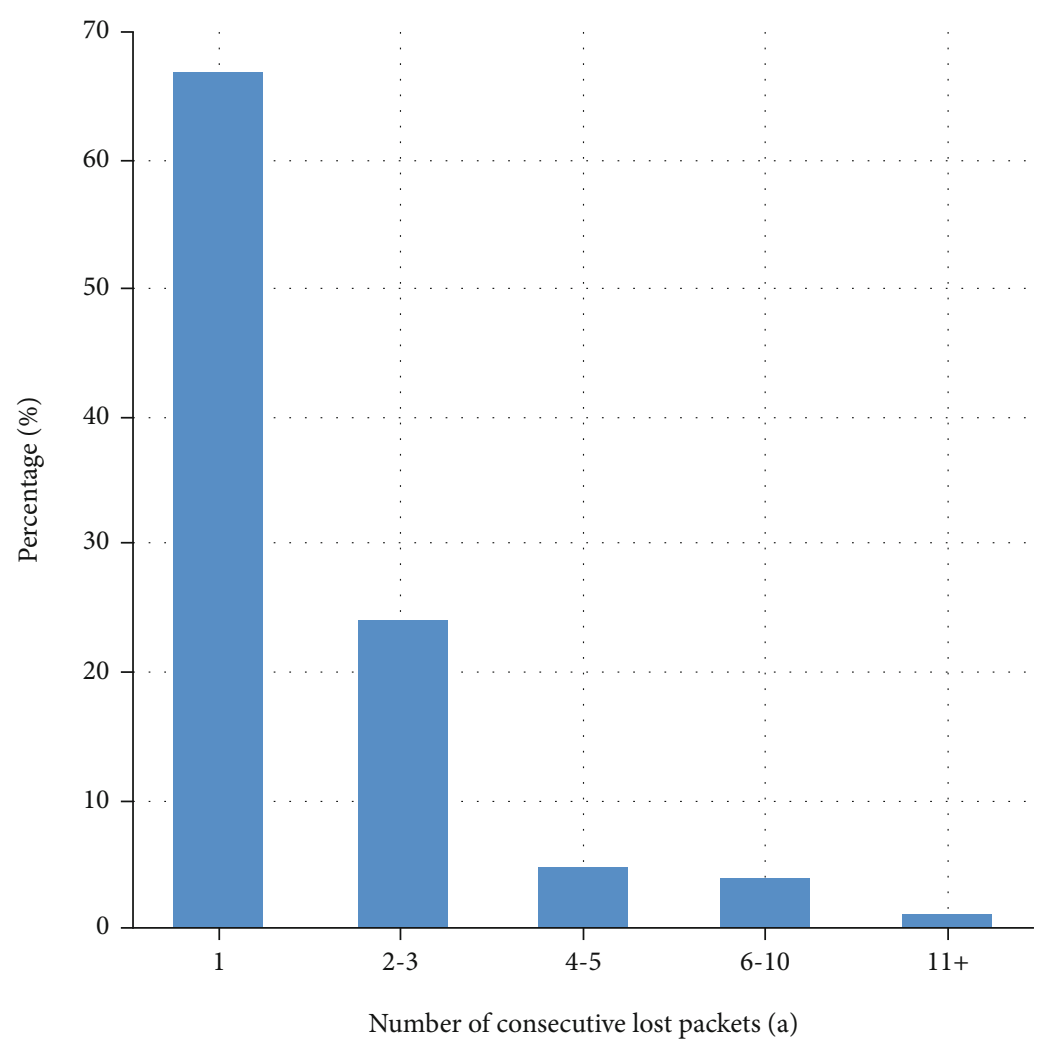

FIGURE 13: Test results for consecutive lost datagrams in the real environment.

for train switching reaches $99.9928 \%$, while when the parameter value is $1050 \mathrm{~ms}$, the reliability of the communication scenario for train reconnection can reach $99.9902 \%$. Simultaneously, the results show that the reconnection process reduces train communication's reliability to a certain extent, affecting the communication between the train and other trains.

As a comparison, consider the reliability of the communication scenario when the train has only one train access unit. According to the simulation results, when the 


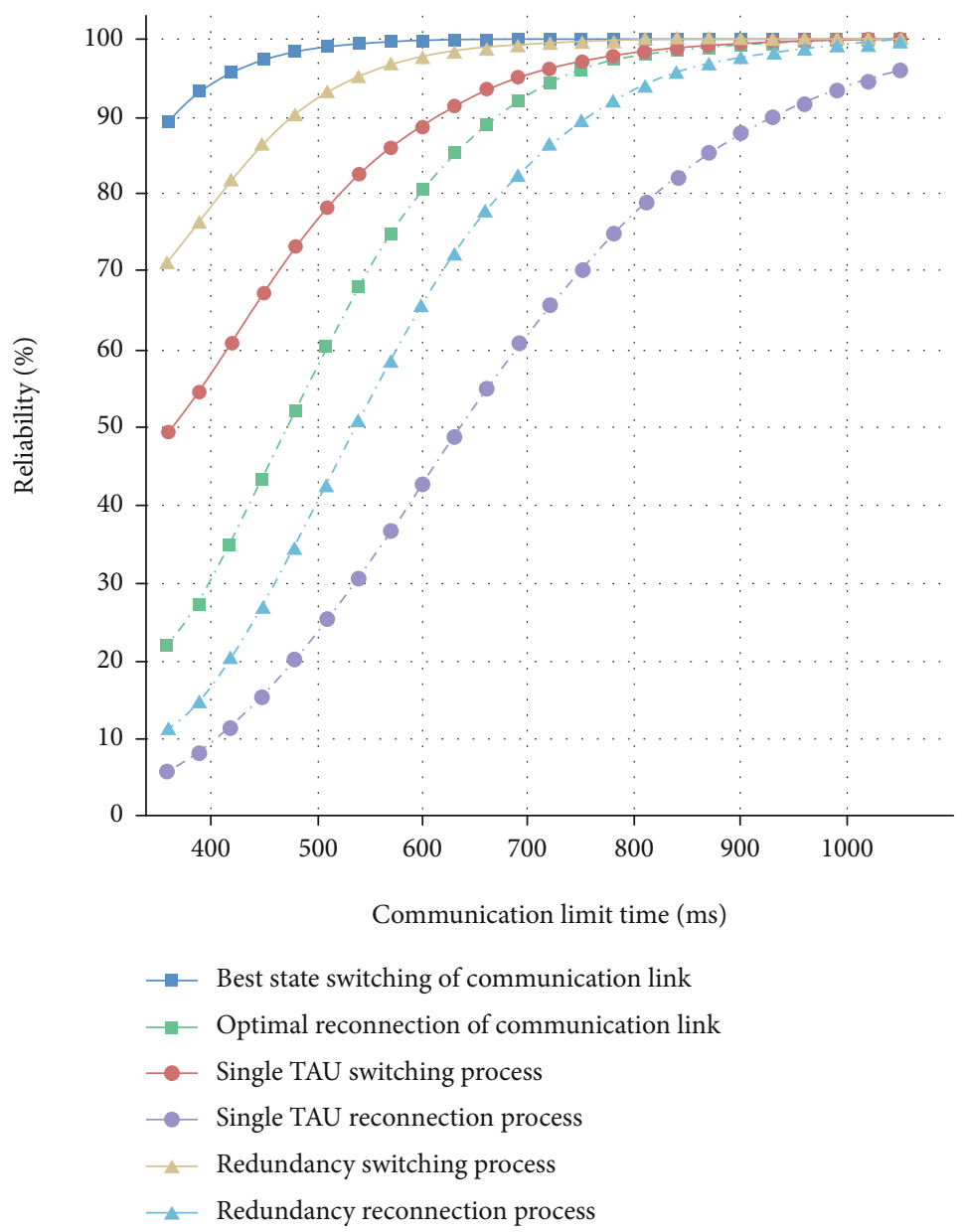

FIgURE 14: The reliability of communication scenarios of redundancy train access units.

communication limit time is short, whether it is a switching process or a reconnection process, the train access unit's reliability is greatly improved when the train access unit is redundant. As it becomes larger, the reliability improvement rate gradually decreases.

Without considering the cable transmission delay and retransmission, the time for handover and reconnection process is related to the wireless transmission delay, and its time conforms to the Gamma distribution, so it can be solved by its density distribution function to complete the wireless under different communication limit time, the probability of transmission. The density function is shown in

$$
f(t)=\frac{t^{\alpha-1} \lambda^{\alpha} e^{-\lambda t}}{(\alpha-1) !}, \quad t>0
$$

where $\alpha$ is the number of signaling that needs to be transmitted using the air interface during the signaling interaction.

In many cases, the reliability of the communication scenario is shown in Figure 14. It can be obtained that the train access unit has better communication reliability when there is redundancy, and its reliability can reach $99 \%$ when the communication limit time is $960 \mathrm{~ms}$, and the reliability exceeds $99.99 \%$ when the communication limit time exceeds
$1050 \mathrm{~ms}$. In the case of nonredundant train access units, the communication limit time needs to be $1230 \mathrm{~ms}$ when the reliability reaches $99 \%$, and when it exceeds $1710 \mathrm{~ms}$, the reliability can exceed $99.99 \%$.

5.2. Reliability Analysis of Communication Scenarios for OnBoard Terminal Handoff. According to the definition of the communication process's reliability, the probability of the train being in the normal state when the parameter value of the transition timeout is different is studied. Therefore, the parameter value of the timeout transition in the top model can be set to a series of values. Under different parameter values, the Monte Carlo method simulates the model to obtain the probability that the train is in the normal state. According to the simulation results, the higher the communication process threshold, the greater the reliability of the communication. When the communication limit time is $1140 \mathrm{~ms}$, the reliability exceeds $99 \%$, and when the communication limit time is $1300 \mathrm{~ms}$, the reliability of the train can reach $99.99 \%$. Simultaneously, with the increase of the communication limit time, the improved range of reliability gradually decreases, indicating that the influence of the communication limit time on reliability gradually decreases. 


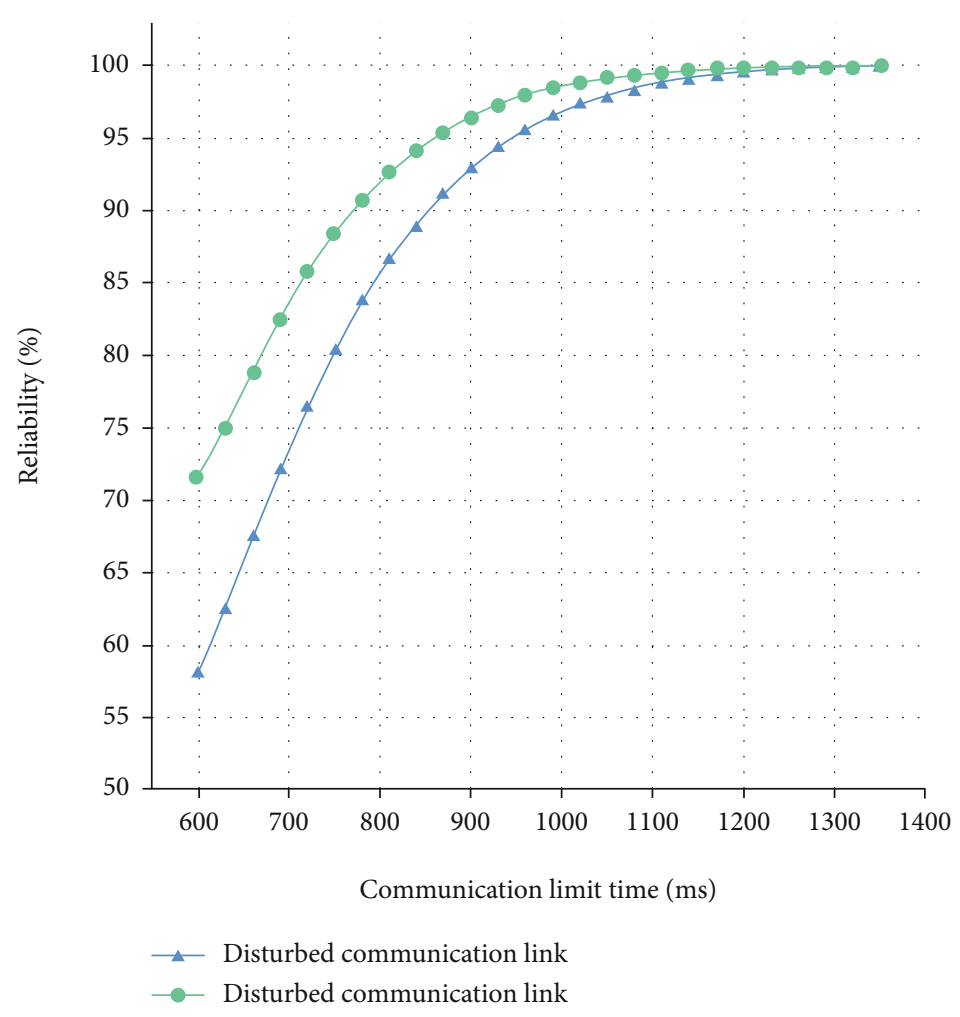

FIgURE 15: The reliability of a communication scenario for on-board terminal handoff.

Without considering the delay and retransmission of cable transmission between ground equipment, the time for the roaming switching communication process conforms to the Gamma distribution, and its density function is Equation (2). Figure 15 shows the reliability of the communication scenario. It can be obtained that when the communication link is in the best state and the communication threshold is less than $1100 \mathrm{~ms}$, the reliability of the communication scenario is higher than the reliability when the link has interference. After the communication limit time gradually increases, the difference in reliability gradually decreases.

\section{Conclusion}

This paper combines the train control DCS based on T2T communication with the existing communication reliability analysis methods to study the reliability of communication scenarios. Based on the structure and principle of the train control system based on T2T communication, the basic structure of the train control DCS based on LTE-U communication technology is studied. The reliability of train control DCS is defined. Simultaneously, two critical communication scenarios of redundancy train access unit and on-board terminal handoff are extracted for the T2T communication scenario, and the communication process is analyzed in detail. Use the $\pi$-tool to build a DSPN model of the scene. Besides, the actual line's communication performance is tested to provide more accurate performance parameters for the model. By setting different communication limit times, the simulation obtained the reliability of crucial communication scenarios. The results show that, combined with the reliabil- ity evaluation method proposed in this paper, DSPN can better realize the communication system models. The reliability research of the DCS in this paper provides an essential reference for the design, implementation, and optimization of the train control DCS based on T2T communication.

\section{Data Availability}

The data used to support the findings of this study are owned by a company.

\section{Conflicts of Interest}

The authors declare that there is no conflict of interest regarding the publication of this paper.

\section{References}

[1] L. Zhu, F. R. Yu, B. Ning, and T. Tang, "Cross-layer handoff design in MIMO-enabled WLANs for communication-based train control (CBTC) systems," IEEE Journal on Selected Areas in Communications, vol. 30, no. 4, pp. 719-728, 2012.

[2] Y. Li, L. Zhu, H. Wang, F. R. Yu, and S. Liu, "A Cross-Layer Defense Scheme for Edge Intelligence-Enabled CBTC Systems Against MitM Attacks," IEEE Transactions on Intelligent Transportation Systems, 2020.

[3] J. Lin, X. Wang, D. Yao, and C. Yan, "Design and research of train collision protection system for urban rail transit," Journal of Railway Science and Engineering, vol. 12, no. 2, pp. 407-413, 2015.

[4] K. Guan, B. Peng, D. He et al., "Channel sounding and ray tracing for train-to-train communications at the $\mathrm{THz}$ 
band $[C] / / 2019$ 13th European Conference on Antennas and Propagation (EuCAP)," IEEE, pp. 1-5, 2019.

[5] L. Jian, "Research on the new generation urban rail transit signal system," Urban Rail Transit Research, vol. 22, no. 7, pp. 71-74, 2019.

[6] H. Wang, F. R. Yu, and H. Jiang, "Modeling of radio channels with leaky coaxial cable for LTE-M based CBTC systems," IEEE Communications Letters, vol. 20, no. 5, pp. 1038-1041, 2016.

[7] C. Luo, F. R. Yu, H. Ji, and V. C. M. Leung, "Cross-Layer Design for TCP Performance Improvement in Cognitive Radio Networks," IEEE Transactions Vehicular Technology, vol. 59, no. 5, pp. 2485-2495, 2010.

[8] Q. Lanhao, "Application of LTE-U in urban rail transit," Urban rapid rail transit, vol. 31, no. 5, pp. 5-10, 2018.

[9] B. Kunai, Research on Wireless Resource and Mobility Management of LTE-U System in Urban Rail Transit, Beijing Jiaotong University, 2019.

[10] L. Zhu, Research on Cross-Layer Design in CBTC Train Ground Communication Systems, Beijing Jiaotong University, 2012.

[11] L. Zhu, Y. He, F. R. Yu, B. Ning, T. Tang, and N. Zhao, "Communication-Based Train Control System Performance Optimization Using Deep Reinforcement Learning," IEEE Transactions Vehicular Technology, vol. 66, no. 12, 2017.

[12] J. Moreno, J. M. Riera, L. de Haro, and C. Rodriguez, “A survey on future railway radio communications services: challenges and opportunities," IEEE Communications Magazine, vol. 53, no. 10, pp. 62-68, 2015.

[13] L. Zhu, Y. Li, F. R. Yu, B. Ning, T. Tang, and X. Wang, "Cross-layer Defense Methods for Jamming-Resistant CBTC Systems," IEEE Transactions on Intelligent Transportation Systems, 2020.

[14] L. Sijia, H. Xiaoge, Z. Fan, and C. Qianbin, "Study on coexistence schemes of LTE-U and WiFi on unlicensed bands," Journal of Chongqing University of Posts and Telecommunications (natural science edition), vol. 29, no. 2, pp. 182-189, 2017.

[15] Z. Luo, LTE-U Network Resource Optimization Based on Machine Learning[D], Zhejiang University, Hangzhou, 2019.

[16] S. Yaqing, DSPN Models and Verification of Vehicle Ground Communication for High Speed Maglev Control System [D], Beijing Jiaotong University, 2013.

[17] M. A. Marsan and G. Chiola, On Petri Nets with Deterministic and Exponentially Distributed Firing Times[C]//European Workshop on Applications and Theory in Petri Nets, Springer, Berlin, Heidelberg, 1986.

[18] W. Peizhong, Design and Simulation of Petri Net Visualization Tool, Xi'an University of Electronic Science and technology, 2012. 